\title{
THE PROOF OF A CONJECTURE OF GRAHAM FOR SEQUENCES CONTAINING PRIMES ${ }^{1}$
}

\author{
RIVKA KLEIN
}

\begin{abstract}
Let $a_{1}<a_{2}<\cdots<a_{n}$ be a finite sequence of positive integers. R. L. Graham has conjectured that $\max _{i, j}\left\{a_{i} /\left(a_{i}, a_{j}\right)\right\} \geq n$. We verify this conjecture in case at least one of the $\alpha_{i}$ 's is prime.
\end{abstract}

R. L. Graham [2] has conjectured that if $a_{1}<a_{2}<\cdots<a_{n}$ is a sequence of positive integers, then $\max _{i, j}\left\{a_{i} /\left(a_{i}, a_{j}\right)\right\} \geq n$. The conjecture has been verified in some special cases. For references see [1]. We mention here (i) the case when $a_{1}$ is a prime [5] and (ii) the case when, for some $k, a_{k}$ is a prime not being of the form $p=\frac{1}{2}\left(a_{i}+a_{j}\right)[\mathbf{4}]$.

In this note we prove Graham's conjecture for sequences containing a prime. Thus we obtain the above result (ii) without any restriction on $p$.

THEOREM. Let $a_{1}<a_{2}<\cdots<a_{n}$ be a sequence of positive integers where $a_{k}=p$, a prime, for some $k$. Then $\max _{i, j}\left\{a_{i} /\left(a_{i}, a_{j}\right)\right\} \geq n$.

PROOF. Assume the contrary that $\max _{i, j}\left\{a_{i} /\left(a_{i}, a_{j}\right)\right\}<n$. Since we may suppose that g.c.d. $\left\{a_{1}, \ldots, a_{n}\right\}=1$, some $a_{i}$ is not a multiple of $p$, so $p=a_{k}=$ $a_{k} /\left(a_{k}, a_{i}\right)<n$. Our sequence contains elements $\geq n$ so $a_{1}>1$. Moreover, each $a_{j} \geq n$ must be a multiple of $p$ since otherwise $a_{j} /\left(a_{j}, a_{k}\right)=a_{j} \geq n$. In particular, if $a_{n}=t p$ then $t=a_{n} /\left(a_{n}, a_{k}\right) \leq n-1$. We claim that $t \leq n-2$. Indeed, by [4, 5], $a_{1}$ is not prime, so $k>1$ and $a_{1}<a_{k}=p$. It follows that $a_{1} \geq 4$ and $p \geq 5$. Since $a_{n} /\left(a_{n}, a_{1}\right) \leq n-1$, we have $a_{n} \leq(n-1) a_{1}$, so $t p \leq(n-1) a_{1}<(n-1) p$ and this implies that $t \leq n-2$.

Consider the following two sets:

$$
A=\{1,2, \ldots, n-1\}, \quad B=\{s p,(s+1) p, \ldots, t p\}
$$

where $s=\lceil n / p\rceil$. By the definitions of $s, t$ and $B$ each $a_{j} \geq n$ belongs to $B$, so $\left\{a_{1}, \ldots, a_{n}\right\} \subseteq A \cup B$.

The proof will be achieved by defining a 1-1 correspondence $F: B \rightarrow A$ such that if $F(b)=a$ then at most one of $a, b$ can be a member of $C=\left\{a_{1}, \ldots, a_{n}\right\}$. Hence $C$ has at most $n-1$ elements, a contradiction.

Let $A^{\prime}=\{c \in A \mid p \nmid c\}$. If $x \in B$ then $x=b p^{r}$ for some $b, p \nmid b$, and $b \leq n-2, r \geq 1$. Define $f: B \rightarrow A^{\prime}$ by $f(x)=b$. The function $f$ is 1-1 for if $f\left(x^{\prime}\right)=f\left(x^{\prime \prime}\right)$ and $x^{\prime} \neq x^{\prime \prime}$, say $x^{\prime \prime}>x^{\prime}$, let $x^{\prime}=b^{\prime} p^{r^{\prime}}, p \nmid b^{\prime}, x^{\prime \prime}=b^{\prime \prime} p^{r^{\prime \prime}}, p \nmid b^{\prime \prime}$. Then $b^{\prime}=f\left(x^{\prime}\right)=$ $f\left(x^{\prime \prime}\right)=b^{\prime \prime}$ and $r^{\prime \prime}>r^{\prime}$ so $x^{\prime \prime}=p^{r^{\prime \prime}-r^{\prime}} x^{\prime} \geq p n>p t$, which is impossible.

Received by the editors November 30, 1984 .

1980 Mathematics Subject Classification. Primary 10A05.

${ }^{1}$ This is a part of the author's Ph.D. Thesis prepared at Tel-Aviv University under the supervision of Professor J. Schönheim. 
Now let $B^{\prime}=f(B)$. Then $B^{\prime} \subset A^{\prime}$. If $b \in B^{\prime}$ then $p \nmid b$ so $b \equiv u(\bmod p)$ for some $u, 1 \leq u \leq p-1$. Define $g: B^{\prime} \rightarrow A^{\prime}$ as follows

$$
g(b)= \begin{cases}b+1 & \text { if } u \text { is odd } \\ b-1 & \text { if } u \text { is even }\end{cases}
$$

The values of $g$ belong to $A^{\prime}$ since $p-1$ is even and $t \leq n-2$. The function $g$ is clearly also $1-1$.

The required correspondence $F$ from $B$ to $A$ is defined by $F(x)=g(f(x))$ and it is clearly $1-1$. We claim that $F(x)$ and $x$ are relatively prime. Indeed, $F(x) \in A^{\prime}$ so $p \nmid F(x)$. In addition, $x=b p^{r}$ and $F(x)=b \pm 1$, so $(b, F(x))=1$, it follows that $\left(b p^{r}, F(x)\right)=1$, thus $(x, F(x))=1$. This implies that $x$ and $F(x)$ cannot both belong to $\left\{a_{1}, \ldots, a_{n}\right\}$ because $x /(x, F(x))=x \geq n$, and this proves the theorem.

\section{REFERENCES}

1. R. D. Boyle, On a problem of R. L. Graham, Acta Arith. 34 (1978), 163-177.

2. R. L. Graham, Unsolved problem 5749, Amer. Math. Monthly 77 (1970), 775.

3. P. Erdös, Problems and results on combinatorial number theory, A Survey of Combinatorial Theory, North-Holland, Amsterdam, 1973, Chapter 12.

4. G. Weinstein, On a conjecture of Graham concerning greatest common divisors, Proc. Amer. Math. Soc. 63 (1977), 33-38.

5. R. Winterle, A problem of R. L. Graham in combinatorial number theory, Proc. Louisiana Conf. on Combinatorics, Graph Theory and Computing, Louisiana State Univ., Baton Rouge, La., $1970,357-361$.

School of Mathematical Sciences, Tel-Aviv University, Tel-Aviv 69978, ISRAEL 